\title{
Denervation does not induce muscle atrophy through oxidative stress
}

Eva Pigna (1), Emanuela Greco (1), Giulio Morozzi (2), Silvia Grottelli (2), Alessio Rotini (3), Alba Minelli (2), Stefania Fulle (3), Sergio Adamo (1)*, Rosa Mancinelli (3), Ilaria Bellezza (2), Viviana Moresi (1)

(1) DAHFMO Unit of Histology and Medical Embryology, Interuniversity Institute of Myology, Sapienza University of Rome, Rome, Italy; (2) Department of Experimental Medicine, University of Perugia, Perugia, Italy; (3) Department of Neuroscience Imaging and Clinical Sciences - Section of Physiology and Physiopathology, University "G. d'Annunzio" ChietiPescara, Chieti, Italy. *Corresponding Author

This article is distributed under the terms of the Creative Commons Attribution Noncommercial License (CC BY-NC 4.0) which permits any noncommercial use, distribution, and reproduction in any medium, provided the original author(s) and source are credited.

\begin{abstract}
Denervation leads to the activation of the catabolic pathways, such as the ubiquitin-proteasome and autophagy, resulting in skeletal muscle atrophy and weakness. Furthermore, denervation induces oxidative stress in skeletal muscle, which is thought to contribute to the induction of skeletal muscle atrophy. Several muscle diseases are characterized by denervation, but the molecular pathways contributing to muscle atrophy have been only partially described. Our study delineates the kinetics of activation of oxidative stress response in skeletal muscle following denervation. Despite the denervation-dependent induction of oxidative stress in skeletal muscle, treatments with anti-oxidant drugs do not prevent the reduction of muscle mass. Our results indicate that, although oxidative stress may contribute to the activation of the response to denervation, it is not responsible by itself of oxidative damage or neurogenic muscle atrophy.
\end{abstract}

Key Words: denervation, oxidative stress, antioxidant drug, neurogenic muscle atrophy.

Eur J Transl Myol 2017; 27 (1): 43-50

Loss of motoneuron innervation severely compromises skeletal muscle mass and function. Neurogenic atrophy is caused by peripheral neuropathies or motor neuron diseases. Muscle atrophy results from increasing catabolism of skeletal muscle proteins often coupled with reduced rates of protein synthesis. ${ }^{1}$ Oxidative stress (OS) has been implicated in many pathological conditions, including sarcopenia ${ }^{2}$, unloading ${ }^{3}$ Amyotrophic lateral sclerosis, ${ }^{4}$ and is now widely considered a major trigger of the imbalance between protein synthesis and degradation. ${ }^{5,6}$ How is OS thought to contribute to muscle atrophy? On one hand ROS directly or indirectly damage mitochondria and cellular components, triggering autophagy, one of the main catabolic pathway. ${ }^{7}$ Changes in mitochondrial shape also activate AMPK, which in turn positively regulates FoxO3, ${ }^{8,9}$ leading to muscle atrophy via the autophagic and ubiquitin-proteasome system. Together with an increase in muscle proteolysis, muscle atrophy is the result of decreased regenerative ability. OS and impaired antioxidant activity negatively affect the differentiation of satellite cells, ${ }^{2,10}$ the muscle progenitor cells, responsible for the repair or growth of adult skeletal muscle. Since excessive OS contributes to muscle damage, a proper response is critical to maintain cell homeostasis. Several transcription factors are activated in response to OS and can trigger either cell protection or cell death, depending on ROS levels. Intracellular oxidative stress is sensed by NF-E2 p45related factor 2 (Nfe2L2), a transcription factor which induces the expression of phase II antioxidant genes. ${ }^{11,12}$ Antioxidant enzymes regulated by Nfe2L2 include: heme oxygenase-1 (Hmox1), NAD(P)H:quinone oxidoreductase (Nqo1), thioredoxin reductase 1 (Txn 1), glutamate-cysteine ligase modifier subunit $(\mathrm{Gclm})$ and glutamate-cysteine ligase catalytic subunit (Gclc). ${ }^{12-14}$ Increased levels of ROS can also be sensed by NFKB. $\mathrm{NF} \kappa \mathrm{B}$ has a dual role in the OS response because, on one hand, NFאB activates several genes that play a major role in antioxidant defense (e.g. manganese superoxide dismutase - MnSOD, Nqo1, glutathione oxidoreductase-1 - Gpx1), on the other hand, other NFkB-targets are involved in ROS generation (e.g. NADPH oxidase $2-$ Cybb, inducible nitric oxide 
synthase - iNOS, cyclooxygenase-2 - Cox2) and skeletal muscle atrophy (MuRF1). Moreover, a cross talk between Nfe2L2 and NF $\kappa$ B has been demonstrated in several experimental models. Indeed, as redoxsensitive transcription factors, depending on the levels of ROS, Nfe2L2 and NFKB are activated, coordinating distinct biological responses. ${ }^{11,12,15,16}$ An increase in mitochondrial-derived reactive oxygen species (ROS) has been reported following denervation, correlating with a decrease in mitochondrial respiration rate, ${ }^{17}$ but its biological relevance has not been addressed yet.

The aim of the present study is to delineate the kinetics of activation of OS and OS-response in murine skeletal muscle following denervation, and to assess the contribution of OS to neurogenic muscle atrophy in vivo.

\section{Material and Methods}

\section{Ethical Approval}

Mice were treated in strict accordance with the guidelines of the Institutional Animal Care and Use Committee and to national and European legislation, throughout the experiments (Permit number 80/2014-B of 03/06/2014). Adult (2.5 month-old) C57BL/6 female mice were used throughout the experiments.

\section{Denervation}

Adult mice were anesthetized, and a $3 \mathrm{~mm}$ segment of the left limb sciatic nerve was excised. The nondenervated contralateral limb was used as control.

When antioxidants were administered, mice were treated daily, starting from the day of denervation, by an intraperitoneal (i.p.) injection of $15 \mathrm{mg} / \mathrm{kg}$ Trolox (Sigma) in $40 \% \mathrm{NaOH}$ in physiologic solution, or 50 $\mathrm{mg} / \mathrm{kg}$ Resveratrol (Sigma) in 1\% EtOH in physiologic solution. Vehicle-treated animals were used as controls.

\section{Histological Analyses}

Tibialis Anterior (TA) muscles were dissected, embedded in tissue freezing medium (Leica, Wetzlar, Germany) and frozen in liquid nitrogen pre-cooled isopentane. Cryosections $(8 \mu \mathrm{m})$ were obtained by using a Leica cryostat. Dihydrohetidium (DHE) staining was performed on transverse cryosections of TA muscles. Unfixed cryosections were incubated with $0,002 \mathrm{mM}$ DHE (Molecular Probes D1168) in DMSO for 30 minutes at $37^{\circ} \mathrm{C}$. Coverslips were mounted with $60 \%$ glycerol in Tris $\mathrm{HCl} 0.2 \mathrm{M} \mathrm{pH} 9.3$. DHE intensity was quantified by measuring the signal in 10 fields for each sample, using image $\mathbf{J}$ software. Hematoxylin and eosin staining was performed according to the Sigma manufacturer's instructions. For immunofluorescences, TA cryosections were fixed in $4 \%$ paraformaldehyde, then permeabilized with $0,2 \%$ triton (Sigma) in phosphate-buffered saline (PBS) and blocked with $10 \%$ BSA (Sigma) in PBS. Samples were then incubated with 1:50 dilution of Nfe2L2 (Santa Cruz (C-20) sc722), followed by incubation with 1:500 dilution of Alexa Fluor® 488 conjugate Secondary Antibody (A11008). Hoechst 33342 (Sigma) was used to stain nuclei.

\section{RNA Extraction and Real-time PCR}

Total RNA was isolated and purified from 30-50 mg of TA muscles by using Trizol (Invitrogen), following the manufacturer's protocol. One microgram of total RNA was converted to cDNA by using the QuantiTect Reverse Transcription Kit (Qiagen). Real-time PCR was performed with the SDS-ABI Prism 7500 (Applied Biosystem), by using the Sybr Green reaction mix (Applied Biosystem). Primers are listed in Table 1.

\section{RNA Extraction and Real-time PCR}

Total RNA was isolated and purified from 30-50 mg of TA muscles by using Trizol (Invitrogen), following the manufacturer's protocol. One microgram of total RNA was converted to cDNA by using the QuantiTect Reverse Transcription Kit (Qiagen). Real-time PCR was performed with the SDS-ABI Prism 7500 (Applied Biosystem), by using the Sybr Green reaction mix (Applied Biosystem). Primers are listed in Table 1.

\section{Protein Extraction and Western Blot Analyses}

TA muscles were dissected, minced, and homogenized in lysis buffer $(50 \mathrm{mM}$ Tris- $\mathrm{HCl} \mathrm{pH} 7.4,1 \mathrm{mM}$ EDTA, $150 \mathrm{Mm} \mathrm{NaCl}, 1 \%$ TRITON) supplemented with

Table 1. Table 1. List of the Primers used in Real-time PCR

\begin{tabular}{|l|l|l|}
\hline Gene & Forward & Reverse \\
\hline$C y b b$ & TCCTATGTTCCTGTACCTTTGTG & GTCCCACCTCCATCTTGAATC \\
\hline$N f e 2 L 2$ & TTGGCAGAGACATTCCCATTTG & AAACTTGCTCCATGTCCTGCTCTA \\
\hline$G c l c$ & GGCGATGTTCTTGAGACTCTGC & TTCCTTCGATCATGTAACTCCC \\
\hline$G c l m$ & CACAGGTAAAACCCAATAGTAACCAAGT & GTGAGTCAGTAGCTGTATGTCAAATTGTT \\
\hline$C y b b$ & TCCTATGTTCCTGTACCTTTGTG & GTCCCACCTCCATCTTGAATC \\
\hline$H m o x l$ & AGCCCCACCAAGTTCAAACA & GCAGTATCTTGCACCA \\
\hline$N q o l$ & CATTCTGAAAGGCTGGTTTGA & CTAGCTTTGATCTGGTTGTCA G \\
\hline$N f e 2 L 2$ & TTGGCAGAGACATTCCCATTTG & AAACTTGCTCCATGTCCTGCTCTA \\
\hline$C a t$ & TGAGAAGCCTAAGAACGCAAT & CCCTTCGCABGCCATGTG \\
\hline$T x n l$ & GTCGTGGTGGACTTCTCTGCTA & TTGTCACAGAGGGAATGGAAGA \\
\hline$g a p d h$ & ACCCAGAAGACTGTGGATGG & CACATTGGGGGTAGGAACAC \\
\hline
\end{tabular}


protease and phosphatase inhibitors (complete Mini EDTA-free and PhosSTOP, ROCHE). Proteins (30-50 micrograms) were separated by SDS-PAGE and transferred to PVDF membrane (Invitrogen). Unspecific binding was blocked in 5\% not fat dry milk in TBST buffer $(20 \mathrm{mM}$ Tris $\mathrm{HCl} \mathrm{pH} 7.6,137 \mathrm{mM} \mathrm{NaCl}, 0.5 \%$ Tween 20). The membranes were incubated overnight, with the appropriate primary antibody diluted in 5\% BSA (Sigma) in TBST. After washing in TBST, membranes were incubated with secondary antibodies HRP-conjugate (Bio-Rad 170-6515 or 170-6516) and signals were detected by using ECL chemistry (Advansta). Images were aquired using films or ChemiDoc MP imaging system (Bio-Rad). Densitometric analyses were performed by measuring band intensity for each sample using Image $\mathbf{J}$ software. The following primary antibodies were used: Gapdh (Santa Cruz sc-32233), Cybb (BD bioscience 611414), P-Rela (Cell Signalling 3033), TOT-Rela (Cell Signalling 4764), 4-HNE (Abcam 46545)

\section{Antioxidant Enzymes Activities}

Muscles were homogenized in $100 \mathrm{mM}$ Na-phosphate buffer $\mathrm{pH} 7.0$ or $\mathrm{pH} 6.5$ depending on the assay, with protease inhibitors cocktail (Sigma) and centrifuged at $100,000 \times \mathrm{g}$ for 15 minutes at $4^{\circ} \mathrm{C}$. Cytosol proteins were measured on the resulting supernatant according to the Bradford's method. Glutathione S-transferase activity was determined by using 1-cloro-2-4dinitrobenzene (CDNB) as substrate. The assay was performed at $340410 \mathrm{~nm}\left(\varepsilon=9.6 \mathrm{mM}^{-1} \mathrm{~cm}^{-1}\right)$ in a final volume of $1 \mathrm{ml}$ containing $100 \mathrm{mM}$ Na-phosphate buffer $\mathrm{pH}$ 6.5, $1 \mathrm{mM}$ CDNB, $1 \mathrm{mM}$ reduced glutathione, and $50 \mu \mathrm{g}$ of protein lysate. Glutathione reductase activity was measured by the rate of decrease in absorbance induced by NADPH oxidation, at $340 \mathrm{~nm}$ $\left(\varepsilon=-6.22 \mathrm{mM}^{-1} \mathrm{~cm}^{-1}\right)$. The assay mixture contained, in a final volume of $1 \mathrm{ml}, 100 \mathrm{mM}$ Na-phosphate buffer $\mathrm{pH} 7.0,1 \mathrm{mM}$ glutathione disulfide, $60 \mu \mathrm{M}$ NADPH and $100 \mathrm{mg}$ of protein lysate.

\section{Protein Carbonyl Assay}

The assay was performed as described in. ${ }^{18}$ The amount of protein-hydrozone produced is quantified spectrophotometrically. Carbonyl content was normalized to protein concentration

\section{Statistics}

Statistical significance was determined using two-tailed Student t-test with a significance level $<0.05$

\section{Results \\ Oxidative Stress is Induced in Skeletal Muscle Following Denervation}

In order to characterize the molecular pathways triggered by denervation in skeletal muscle, we severed the sciatic nerve of one limb of adult mice and analyzed the skeletal muscle response over time. To reduce the number of mice, we first tested if contralateral innervated muscles could be used as control. Oxidative stress was evaluated by measuring the ROS levels by Dihydrohetidium (DHE) staining and the expression levels of cytochrome $b-245$, beta polypeptide $(C y b b)$ in the contralateral TA muscles, compared with control mice, not subjected to denervation. No significant differences were observed between the two groups (Fig. 1), allowing contralateral muscles to be used as control muscles, thus reducing the number of experimental animals

To assess if denervation induces OS in skeletal muscle, ROS were labeled by DHE in contralateral and in denervated muscles. Denervated muscles showed a time-dependent increase in ROS levels, compared to contralateral muscles (Fig. 2A). This result was confirmed by the kinetic of induction of $C y b b$ expression, at both the mRNA and protein levels, (Fig. $2 \mathrm{~B}$ and $\mathrm{C}$ ). OS activates NF $\mathrm{B}$ which, in turn, regulates the expression of $C y b b$. To evaluate if denervation induces NFkB activation, the levels of the phosphorylated, active form of the Rela subunit (PRela) of NFKB were monitored in TA muscles, over
A

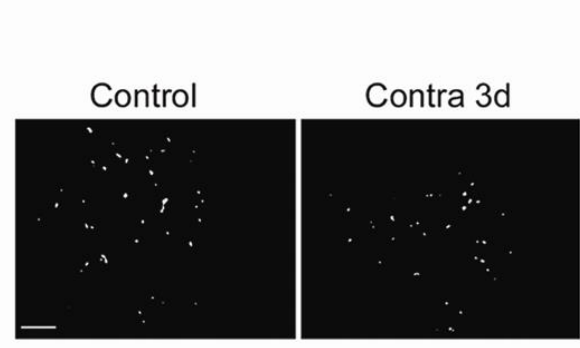

B

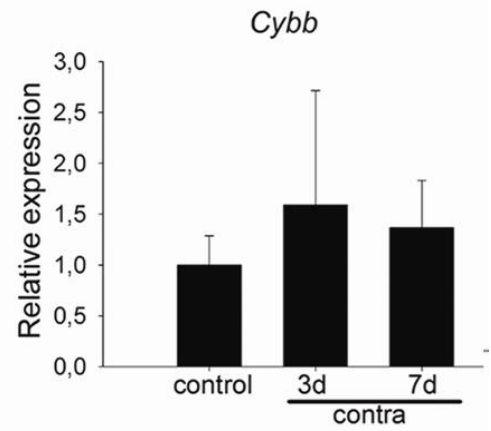

Fig 1. Oxidative stress is not induced in non-denervated, contralateral muscles. (A) DHE staining in TA of mice not subjected to denervation (control) or contralateral muscles of denervated mice (contra), 3 and 7 days following denervation. Scale bar $=50 \mu \mathrm{m}$. (B) Real-time PCR of $C y b b$ in control and contralateral muscles, 3 and 7 days following denervation. Values represent mean \pm SEM. $n=3$. 


\section{Muscle denervation atrophy is not related to oxidative stress}

Eur J Transl Myol 2017; 27 (1): 43-50

time. NFאB activation was detected as early as three days following denervation, and it further increased seven days after denervation (Fig. 2D).

Since NFKB cross-talks with the Nfe2L2, we monitored
Nfe2L2 activation in skeletal muscle upon denervation. Real-time PCR analyses showed increased levels of $N f e 2 L 2$ expression at three and seven days following denervation (Fig. 2E). Importantly, Nfe2L2 mainly

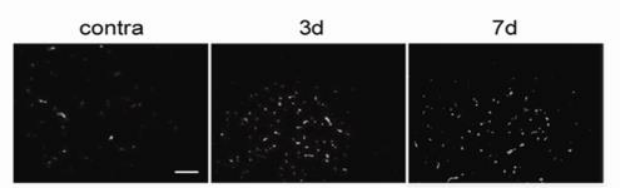

C
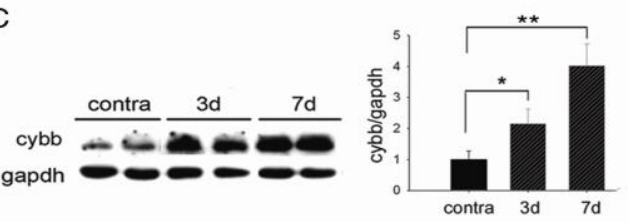

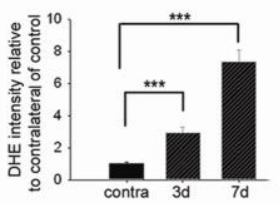

B

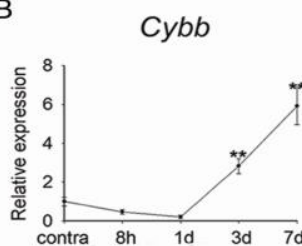

D
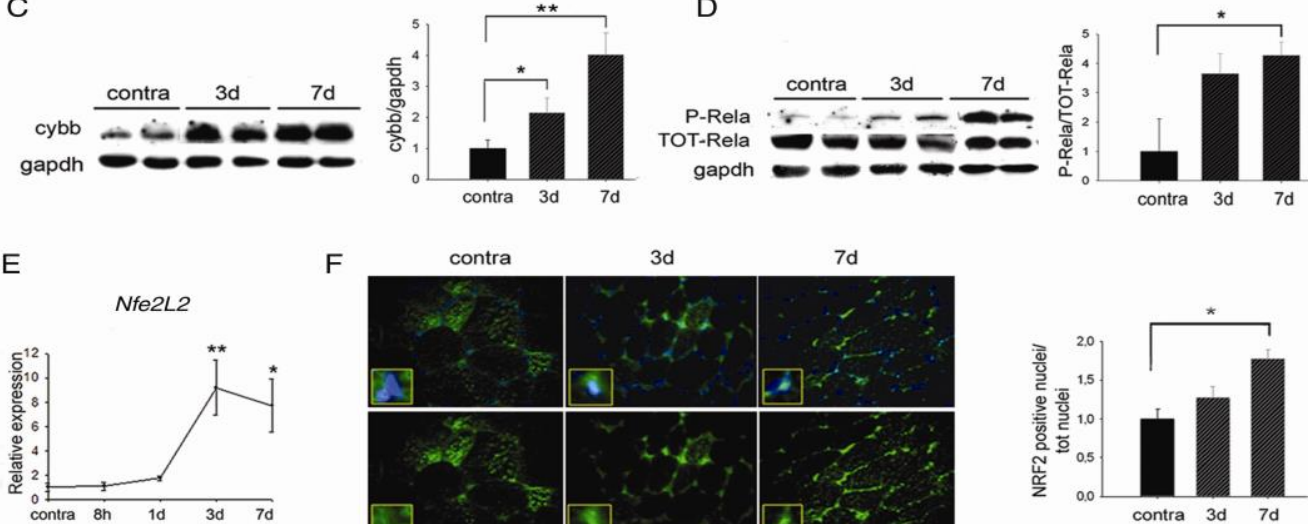

$\mathrm{F}$ contra $3 d$ $7 d$
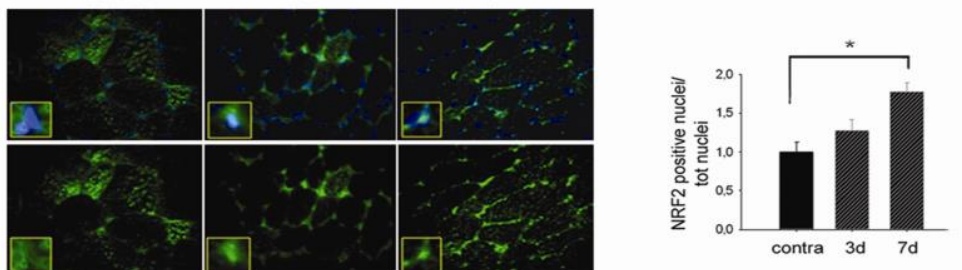

G

Gclc

Gclm

$\mathrm{H}$

Gsr
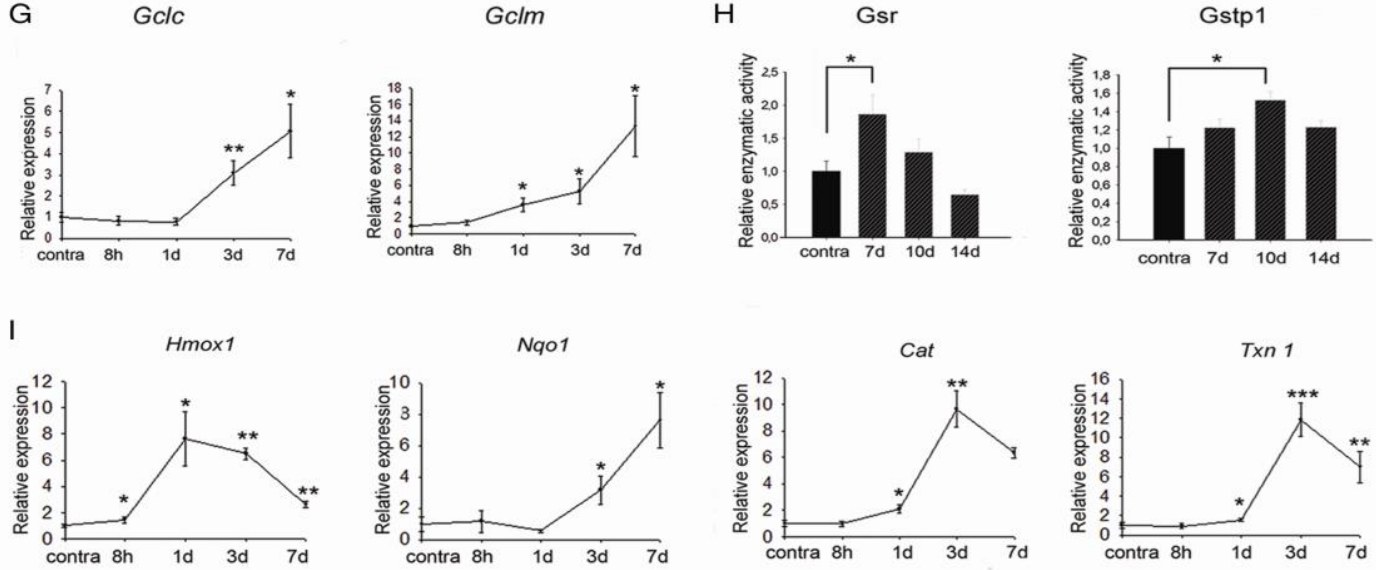

Fig 2. Oxidative stress is induced in skeletal muscle following denervation. (A) DHE staining and quantification in innervated (contra) or denervated TA muscles, 3 and 7 days following denervation. Scale bar $=50 \mu \mathrm{m}$. Values represent mean \pm SEM. $n$ $=4$. ${ }^{*} * \mathrm{p}<0.0001$. (B) Real-time PCR for $C y b b$ at different time points following denervation, relative to innervated contralateral muscles. Values represent mean \pm SEM. $n=4-6$. $* p<0.05 ; * * p<0.001$ vs contralateral muscles. (C) Western blotting analyses and densitometry of Cybb at indicated time points following denervation. Gapdh was used as loading control. $\mathrm{n}=2-4 ;{ }^{*} \mathrm{p}<0.05$. (D) Western blot analyses and densitometry of $\mathrm{P}$ - and total Rela subunit of NFKB in TA muscles at the indicated time points following denervation. Gapdh was used as loading control. $n=2-4$; ${ }^{*}<0.05$. (E) Real-time PCR for $N f e 2 L 2$ in TA muscles at indicated time points following denervation, respect to contralateral muscles. Values represent mean \pm SEM. $\mathrm{n}=4-6 .{ }^{*} \mathrm{p}<0.05 ; * * \mathrm{p}<0.001$ vs contra muscles. (F) Immunofluorescence staining and quantification of intracellular localization of Nfe2L2 in skeletal muscle following denervation. Scale bar $=20 \mu \mathrm{m}$. Values represent mean \pm SEM. $\mathrm{n}=2$ each time point. $* \mathrm{p}<0.05$. (G) Real-time PCR of Gclc and Gclm at indicated time points following denervation. Values represent mean \pm SEM. $n=4-6 .{ }^{*} \mathrm{p}<0.05 ; * * \mathrm{p}<0.001$ vs contra muscles. $(\mathrm{H})$ Enzymatic activities of Gsr and Gstp1 at indicated time points following denervation, relative to contralateral muscles. $n=4$. Values represent mean \pm SEM *p $<0.05$ vs contra muscles. (I) Real-time PCR of indicated genesat indicated time points following denervation, relative to contralateral muscles. Values represent mean \pm SEM. $\mathrm{n}=4-6 . *_{\mathrm{b}}<0.05: * * \mathrm{~b}<0.001$ vs contra muscles. 
translocated into the nuclei at three and, even more, at seven days following denervation, compared to contralateral muscles (Fig. 2F). To confirm the kinetics of Nfe2L2 activation, we evaluated the expression of glutamate-cysteine ligase catalytic $(G c l c)$ and modifier $(G c l m)$ subunits, two known Nfe2L2 target genes ${ }^{14}$ (Fig. $2 \mathrm{G})$. The expression of both genes increased over time following denervation, confirming that Nfe2L2 is more active at seven than at three days following denervation. Glutathione plays a key role in cellular detoxification processes $^{19}$ through glutathione reductase (Gsr) and glutathione S-transferase pi 1 (Gstp1) enzymes. Gsr activity was significantly increased at seven days, but it decreased at ten and fourteen days after denervation; conversely, Gstp1 achieved its maximal activity ten days following denervation (Fig. $2 \mathrm{H}$ ).

To determine the molecular response of skeletal muscle to OS, we monitored the expression of several genes involved in ROS buffering, over time. A significant induction of Hmoxl expression was detected as soon as eight hours following denervation reached the maximal expression at one day and decreased after three days. Expression of $\mathrm{Nqol}$ gene increased in a time-dependent manner, beginning at day three. The expression of other genes - i.e. catalase (Cat) and thioredoxin 1(Txn 1) showed a significant induction one day following denervation, reached a peak after three days and decreased seven days after denervation (Fig. $2 \mathrm{H}$ ).

The effects of ROS are dose-dependent since they can act as physiological signaling molecules at low levels but they can be harmful above a threshold. ${ }^{20}$ To verify if the induction of OS upon denervation leads to muscle damage, protein carbonyl level and lipid peroxidation, sensitive biomarkers of ROS-mediated damage, were quantified in skeletal muscle tissue, seven days following denervation. Interestingly, denervation induced neither a significant increase in carbonyl groups nor an induction in lipid peroxidation (Fig. 3), as detected by 4-Hydroxynonenal (4-HNE) levels, suggesting that skeletal muscle is not damaged by OS after severing nerve.

\section{Oxidative Stress Does not Induce Muscle Atrophy Following Denervation}

Denervation induces skeletal muscle atrophy and OS. To investigate the contribution of OS to neurogenic muscle atrophy, mice were treated with Trolox, a cellpermeable derivate of vitamin $\mathrm{E}$ with antioxidant properties, ${ }^{21,22}$ or vehicle as a control, starting from the day of denervation. We first verified that Trolox treatment effectively reduced OS in skeletal muscle following denervation, by evaluating cybb levels by Western blot analyses (Fig. 4A). Muscle mass evaluation and histological analyses showed that Trolox exerted no protective effects on muscle atrophy, two and four weeks following denervation (Fig. 4B \& 4C). Similar results were obtain by treating mice with another anti-oxidant drug, Resveratrol, ${ }^{23}$ for four weeks
A

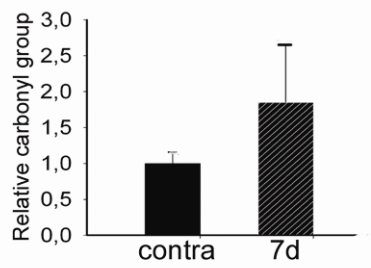

B

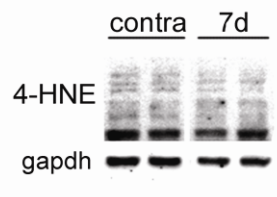

Fig 3. Oxidative stress does not induce cellular damage in skeletal muscle following denervation. (A) Protein carbonyl content relative to contralateral muscles, seven days following denervation. $\mathrm{n}=3$. (B) Western blotting analyses for 4-HNE in contralateral and denervated muscles, 7 days following denervation. Gapdh was used as loading control.

following denervation (data not shown). These results indicate that denervation activates OS, which is however not responsible for neurogenic muscle atrophy.

\section{Discussion}

In this study, we examined the kinetics of activation of the OS response following denervation in skeletal muscle, and determined if ROS activation plays a causal role in the causing muscle atrophy. We showed that OS is induced in skeletal muscle as early as three days following denervation, prior to the appearance of muscle atrophy. Consistently, the induction of antioxidant response genes began at day three and increased over time following denervation, as confirmed by nuclear translocation of Nfe2L2 and by the kinetics of expression of Gclc and Gclm genes, two Nfe2L2 targets. It has been reported that muscle inactivity or denervation increases the levels of ROS as a consequence of both reduced antioxidant capacity and induced production of ROS. ${ }^{24-26}$ Furthermore, exercise and Functional Electrical Stimulation (FES) greatly improve muscle mass and functionality in denervated patients; ${ }^{27,28}$ although, a direct correlation between treatment and improved antioxidant defense has established only for physical activity, while FES stimulus is insufficient to change oxidative status on denervated muscles. ${ }^{29,30}$ Here we showed that the expression of antioxidant genes increased quickly following denervation, suggesting that $\mathrm{OS}$ is due to ROS generation rather than to a reduced antioxidant response. Moreover, distinct antioxidant genes are induced at different time points and with different kinetics, probably due to the different substrate specificity of the encoded enzymes. ${ }^{31}$

The principal source of ROS in eukaryotic cells are mitochondria. Denervation induces a rapid decrease in muscle mitochondrial content ${ }^{32}$ and an unbalance in mitochondrial ROS production. ${ }^{33}$ Moreover, numerous enzymes can also generate ROS, including NADPH oxidases, cyclooxygenases, lipoxygenases and NO synthases. ${ }^{34}$ We found an increase in the expression of 
A
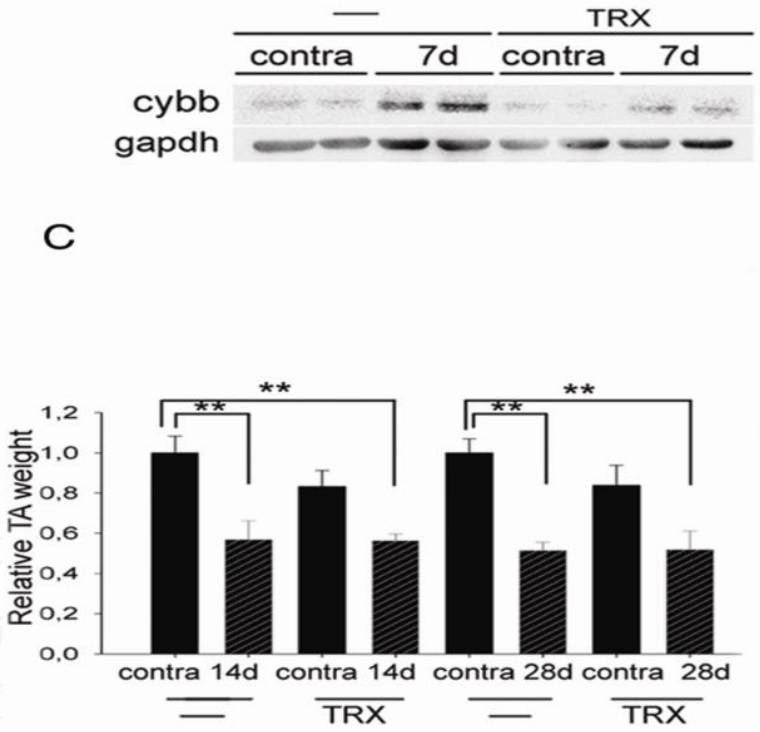

B

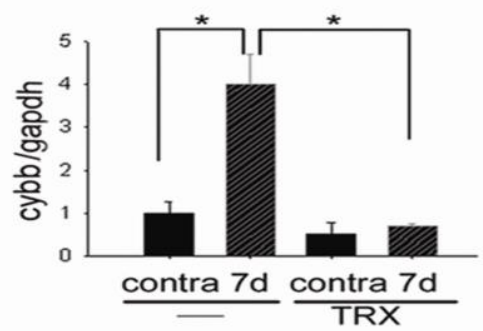

D

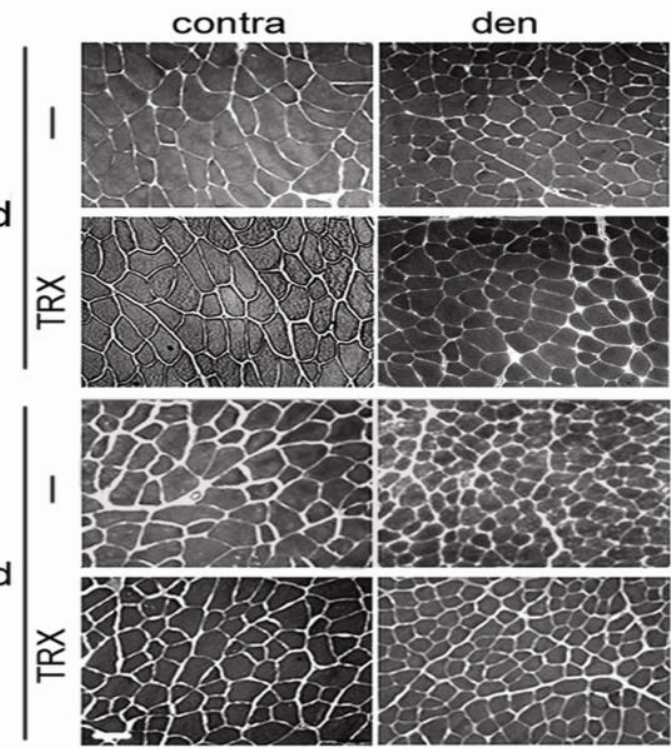

Fig 4. Trolox does not prevent neurogenic muscle atrophy. (A) Western blotting analyses and (B) densitometry of Cybb of vehicle (-) or Trolox (TRX) treated mice, 7 days following denervation. Gapdh was used as loading control. Values represent mean \pm SEM. $n=2-4 ; * p<0.05$. (C) TA weight of vehicle or Trolox treated contralateral and denervated muscles, 14 and 28 days following denervation, relative to contralateral muscles of vehicle-treated mice. Values represent mean \pm SEM. $\mathrm{n}=3$. ${ }^{* *} \mathrm{p}<0.01$. (D) Representative histology of vehicle or Trolox treated contralateral and denervated muscles, 14 and 28 days following denervation. Scale bar $=50 \mu \mathrm{m}$.

$C y b b$, a subunit of NADPH oxidase, starting at three days after denervation, accompanied by a timedependent increase in NFkB activation. These findings suggest that $\mathrm{NF \kappa B}$ might be responsible, at least in part, for ROS generation in denervated muscles. While there was a clear activation of the response to OS, no OSdependent damage was detected upon denervation, such as high levels of carbonyl groups and lipid peroxidation. Therefore, it seems likely that denervation triggers OS above the "harmful threshold" in skeletal muscle. ${ }^{20}$

To test if OS play a direct role in the induction of muscle atrophy, we treated mice with antioxidant drugs, Trolox or Resveratrol, daily, for four weeks after denervation. Although both treatments reduced OS levels in skeletal muscle, the reduction of muscle mass was not affected by the drugs, indicating that neurogenic muscle atrophy does not depend on OS. Similar conclusions were drawn from several studies performed on murine models of disuse muscle atrophy, where Trolox treatment failed to protect against muscle mass loss. ${ }^{35-37}$ In conclusion, our study clarifies the kinetics of activation of OS response in denervated skeletal muscle. Despite the increase, OS does not lead to skeletal muscle damage and does not cause muscle atrophy in response to denervation. Delineation of the kinetics of activation of the molecular pathways triggered by the loss of motor neurons and their relative importance in the activation of the catabolic pathways, responsible of skeletal muscle atrophy, is a prerequisite for developing more appropriate drugs to treat neurogenic muscle atrophy.

\section{Author's contributions}

Conception of the work: VM, IB. Acquisition, analysis, and interpretation of data: EP, EG, GM, SG, AR, RM, SA, IB and VM. Drafting the manuscript and critically revising it for important intellectual content: EP, EG, VM, SA, RM, AM, SF and IB. All authors approved the final version of the manuscript and agree to be accountable for all aspects of the work in ensuring that questions related to the accuracy or integrity of any part of the work are appropriately investigated and resolved. 
All persons designated as authors qualify for authorship, and all those who qualify for authorship are listed.

\section{Acknowledgments}

This work was supported by FIRB 2012 grant (RBFR12 BUMH) and PRIN 2012 grant (2012N8YJC3) from MIUR. We thank Carla Ramina for technical assistance with the experimental analyses.

\section{Conflict of Interest}

The authors submit no conflict of interest regarding the publication of this article.

\section{Corresponding Author}

Sergio Adamo

Via Antonio Scarpa 16, 00161 Roma

$\mathrm{T}(+39) 0649766756$ (26756) / 3492682502

F (+39) 064462854

Email: sergio.adamo@uniroma1.it

\section{E-mails of coAuthors}

Eva Pigna: eva.pigna@uniroma1.it

Emanuela Greco: emanuela.greco1505@gmail.com

Giulio Morozzi: morozzigiulio@gmail.com

Silvia Grottelli: silvia.grottelli@unipg.it

Alessio Rotini: alessio.rotini@gmail.com

Alba Minelli: alba.minelli@unipg.it

Stefania Fulle: sfulle@ unich.it

Rosa Mancinelli: r.mancinelli@unich.it

Ilaria Bellezza: ilaria.bellezza@unipg.it

Viviana Moresi: viviana.moresi@uniroma1.it

\section{References}

1. Glass DJ. Molecular mechanisms modulating muscle mass. Trends Mol Med 2003;9:344-350.

2. Fulle S, Di DS, Puglielli C, Age-dependent imbalance of the antioxidative system in human satellite cells. Exp Gerontol 2005; 189-197.

3. Powers SK, Kavazis AN, DeRuisseau KC. Mechanisms of disuse muscle atrophy: role of oxidative stress. Am J Physiol Regul Integr Comp Physiol 2005; R337-R344.

4. FL Muller, W Song, YC Jang, et al. Denervationinduced skeletal muscle atrophy is associated with increased mitochondrial ROS production. Am J Physiol Regul Integr Comp Physiol 2007;293:R1159-R1168.

5. Moylan JS, Reid MB. Oxidative stress, chronic disease, and muscle wasting. Muscle Nerve 2007; 411-429.

6. Powers SK, Duarte J, Kavazis AN, Talbert EE. Reactive oxygen species are signalling molecules for skeletal muscle adaptation. Exp Physiol 2010; 1-9.

7. Sandri, M. Autophagy in skeletal muscle. FEBS Lett. 2010; 1411-1416.

8. Nakashima,K. \& Yakabe,Y. AMPK activation stimulates myofibrillar protein degradation and expression of atrophy-related ubiquitin ligases by increasing FOXO transcription factors in $\mathrm{C} 2 \mathrm{C} 12$ myotubes. Biosci. Biotechnol. Biochem 2007; 1650-1656.

9. Romanello V, Guadagnin E, Gomes L, et al. Mitochondrial fission and remodelling contributes to muscle atrophy. EMBO J 2010; 1774-1785.

10. Beccafico S, Puglielli C, Pietrangelo T, et al. Agedependent effects on functional aspects in human satellite cells. Ann N Y Acad Sci 2007; 345-352.

11. Bellezza I, Mierla AL, Minelli A. Nrf2 and NFkappaB and Their Concerted Modulation in Cancer Pathogenesis and Progression. Cancers (Basel) 2010;483-497.

12. Minelli A, Conte C, Grottelli S, Bellezza I, et al. Cyclo(His-Pro) promotes cytoprotection by activating Nrf2-mediated up-regulation of antioxidant defence. J Cell Mol Med 2009; 11491161.

13. Minelli A, Bellezza I, Tucci A. Differential involvement of reactive oxygen species and nucleoside transporters in cytotoxicity induced by two adenosine analogues in human prostate cancer cells. Prostate 2009; 538-547.

14. Niture SK, Khatri R, Jaiswal AK. Regulation of Nrf2-an update. Free Radic Biol Med 2014; 36-44.

15. Abdelsalam RM, Safar MM. Neuroprotective effects of vildagliptin in rat rotenone Parkinson's disease model: role of RAGE-NFkappaB and Nrf2-antioxidant signaling pathways. J Neurochem 2015; 700-707.

16. Huang CS, Lin AH, Yang TC, Liu KL, Chen HW et al. Shikonin inhibits oxidized LDL-induced monocyte adhesion by suppressing NFkappaB activation via up-regulation of $\mathrm{PI} 3 \mathrm{~K} / \mathrm{Akt} / \mathrm{Nrf2}$ dependent antioxidation in EA.hy926 endothelial cells. Biochem Pharmacol 2015; 352-361.

17. O'Leary MF, Hood DA. Denervation-induced oxidative stress and autophagy signaling in muscle. Autophagy 2009; 230-231.

18. Zusterzeel PL, Mulder TP, Peters WH, et al. Plasma protein carbonyls in nonpregnant, healthy pregnant and preeclamptic women. Free Radic. Res. 2000; 471-476.

19. Limon-Pacheco J, Gonsebatt ME. The role of antioxidants and antioxidant-related enzymes in protective responses to environmentally induced oxidative stress. Mutat Res 2009; 137-147.

20. Musaro A, Fulle S, Fano G. Oxidative stress and muscle homeostasis. Curr Opin Clin Nutr Metab Care 2010; 236-242.

21. Salgo MG, Pryor WA. Trolox inhibits peroxynitrite-mediated oxidative stress and apoptosis in rat thymocytes. Arch Biochem Biophys 1996; 482-488.

22. Wu TW, Hashimoto N, Wu J, Carey D, et al. The cytoprotective effect of Trolox demonstrated with three types of human cells. Biochem Cell Biol 1990; 1189-1194. 


\section{Muscle denervation atrophy is not related to oxidative stress}

Eur J Transl Myol 2017; 27 (1): 43-50

23. Lancon A, Frazzi R, Latruffe N. Anti-Oxidant, Anti-Inflammatory and Anti-Angiogenic Properties of Resveratrol in Ocular Diseases. Molecules 2016;21:304.

24. Powers SK, Smuder AJ, Judge AR. Oxidative stress and disuse muscle atrophy: cause or consequence? Curr Opin Clin Nutr Metab Care 2012; 240-245.

25. Carraro U, Coletti D, Kern H. The Ejtm Special "The Long-Term Denervated Muscle". Eur J Transl Myol 2014;24:73.

26. Abruzzo PM, di Tullio S, Marchionni C, et al. Oxidative stress in the denervated muscle. Free Radic Res 2010;44:563-76.

27. Carraro U, Kern H, Gava P et al. Biology of muscle atrophy and of its recovery by FES in aging and mobiliting impairments: Roots and ByProducts. Eur J Transl Myol 2015;25:221-30.

28. Kern H, Carraro U. Home-Based Functional Electrical Stimulation For Long-Term Denervated Human Muscle: History, Basics, Results and Perspectives Of The Vienna Rehabilitation Strategy. Eur J Transl Myol 2014;24:3296

29. Ordonez FJ, Roseti MA, Camacho A et al. Armcrancking exercise reduced oxidative damage in adults with chronic spinal cord injury. Arch Phys Med Rehabil 2013;94: 2336-41

30. Van Duijnhoven N, Hesse E, Janssen $\mathrm{T}$ et al. Impact of exercise training on oxidative stress in individuals with a spinal cord injury. Eur J Appl Physiol 2010;109:1059-66
31. Morgan MJ, Liu ZG. Crosstalk of reactive oxygen species and NF-kappaB signaling. Cell Res 2011; 103-115.

32. Tryon LD, Crilly MJ, Hood DA. Effect of denervation on the regulation of mitochondrial transcription factor A expression in skeletal muscle. Am J Physiol Cell Physiol 15-8-2015; C228-C238.

33. Adhihetty PJ, O'Leary MF, Chabi B, et al. Effect of denervation on mitochondrially mediated apoptosis in skeletal muscle. J. Appl. Physiol (1985.) 2007; 1143-1151.

34. Reid MB. Invited Review: redox modulation of skeletal muscle contraction: what we know and what we don't. J Appl Physiol (1985 ) 2001; 724731.

35. Brocca L, Pellegrino MA, Desaphy JF, et al. Is oxidative stress a cause or consequence of disuse muscle atrophy in mice? A proteomic approach in hindlimb-unloaded mice. Exp Physiol 2010;331350 .

36. Cannavino J, Brocca L, Sandri M, Bottinelli R, et al. PGC1-alpha over-expression prevents metabolic alterations and soleus muscle atrophy in hindlimb unloaded mice. J Physiol 2014; 45754589.

37. Desaphy JF, Pierno S, Liantonio A, et al. Antioxidant treatment of hindlimb-unloaded mouse counteracts fiber type transition but not atrophy of disused muscles. Pharmacol Res 2010; 553-563. 